\title{
Incremental Updating of Rough Approximations in Interval-valued Information Systems under Attribute Generalization ${ }^{\text {th }}$
}

\author{
Yingying Zhang ${ }^{\mathrm{a}}$, Tianrui Li ${ }^{\mathrm{a}, *}$, Chuan Luo ${ }^{\mathrm{b}}$, Junbo Zhang ${ }^{\mathrm{a}}$, Hongmei Chen ${ }^{\mathrm{a}}$ \\ ${ }^{a}$ School of Information Science and Technology, Southwest Jiaotong University, Chengdu 611756, China \\ ${ }^{b}$ College of Computer Science, Sichuan University, Chengdu 610065, China
}

\begin{abstract}
Interval-valued Information System (IvIS) is a generalized model of single-valued information system, in which the attribute values of objects are all interval values instead of single values. The attribute set in IvIS is not static but rather dynamically changing over time with the collection of new information, which results in the continuous updating of rough approximations for rough set-based data analysis. In this paper, on the basis of the similarity-based rough set model in IvIS, we develop incremental approaches for updating rough approximations in IvIS under attribute generalization, which refers to the dynamic changing of attributes. Firstly, increment relationships between the original rough approximations and the updated ones when adding or deleting an attribute set are analyzed, respectively. And the incremental mechanisms for updating rough approximations in IvIS are introduced, which carry out the computation using the previous results from the original data set along with new results. Then, the corresponding incremental algorithms are designed based on the proposed mechanisms. Finally, comparative experiments on data sets from UCI as well as artificial data sets are conducted, respectively. Experimental results show that the proposed incremental algorithms can effectively reduce the running time for the computation of rough approximations in comparison with the static algorithm.
\end{abstract}

Keywords: Interval-valued information system, Similarity degree, Rough set, Incremental updating, Approximations.

\section{Introduction}

Rough Set Theory (RST), introduced by Pawlak [25], is an effective mathematical tool to deal with imprecise, vague and uncertain data without any external information. The uncertain concept and knowledge hidden in an information system can be described by the lower and upper approximations in RST. Nowadays, many rough set based data analysis approaches have been successfully applied in medical diagnosis [12], financial analysis [4, 39], industrial control [32], investment decisions [1, 29,30] and many other fields.

Data analyzed by RST are commonly interpreted and presented through the information system, which includes objects, features (attributes) and attribute values [26]. The classical RST can only deal with single-valued information systems with certain category attributes by using an indiscernibility relation. Information in the real world, however, is complex. Many unexpected factors like measurement errors lead to the existence of imprecision. And the attribute values in an information system are often represented in the form of interval numbers, which are not single values but sets of numbers ranging from a minimum to a maximum value. For example, tolerance limits in quality control, financial data (opening value and closing value in a session) and customer satisfaction data (expected or perceived characteristic of the quality of a product) [13]. Interval algebra provides us a very powerful and valid tool for data

\footnotetext{
This is an extended version of the paper presented at the 2015 International Conference on Rough Sets and Knowledge Technology (RSKT2015), Tianjin, 2015, China.

${ }^{*}$ Corresponding author.

Email addresses: yingzhang86@126.com (Yingying Zhang), trli@swjtu.edu.cn (Tianrui Li ), cluo@scu.edu.cn (Chuan Luo), jbzhang@my.swjtu.edu.cn (Junbo Zhang), hmchen@swjtu.edu.cn (Hongmei Chen)
} 
analysis since it was put forward by Moore [22, 23, 40-42]. It has been widely applied in data mining [9, 24], computational intelligence $[28,36]$ and multi-attribute decision making problems $[2,33,35]$. The information system whose attribute values are all intervals is called as Interval-valued Information System (IvIS).

In recent years, many extensions of classical RST have been applied in IvIS based on different binary relations. By introducing the concept of misclassification rate between two interval numbers, Leung et al. proposed a tolerancebased rough set model for extracting classification rules from IvIS [14]. Based on the similarity rate between two interval values, Miao et al. extended the concept of original maximal consistent blocks to formulate the rough approximations in IvIS [21]. In virtue of interval-inclusion degree, Zhang et al. introduced a binary neighborhood relation on an IvIS as well as the concept of interval-valued granular rules and proposed a confidence-preserved attribute reduction approach to extract compact decision rules [44]. Based on the different definitions of the degree that an interval number is dominating another one, different dominance-based rough set models were introduced into IvIS to attribute reduction and rule extraction [10, 27, 37, 38]. Dai et al. addressed the uncertainty measurement problem in IvIS by introducing the concepts of possible-degree-based conditional entropy and similarity relation between two interval values, respectively [7, 8]. Zhang et al. proposed a variable-precision-dominance-based rough set model with inclusion measure theory in IvIS [45]. Sun et al. introduced the interval-valued fuzzy rough set model into intervalvalued fuzzy information systems, in which the binary relation is an interval-valued fuzzy relation [31]. Zhang et al. proposed a general study of interval-valued fuzzy rough sets on two universes of discourse integrating the rough set theory with the interval-valued fuzzy set theory by constructive and axiomatic approaches [49]. Considering the value of loss function with the imprecise evaluation, Liang et al. explored the interval-valued decision-theoretic rough sets [17]. By integrating fuzzy probability measure and interval-valued fuzzy probability measure into Bayesian decision procedure, Zhao et al. introduced fuzzy decision-theoretic rough set (FDTRS) approach and interval-valued fuzzy decision-theoretic rough set (IVF-DTRS) approach to directly deal with real-valued and interval-valued data [50]. By ranking any two interval-valued intuitionistic fuzzy values, Huang et al. introduced a dominance relation in the framework of interval-valued intuitionistic fuzzy information systems and established a dominance-based rough set model to simplify knowledge representation and extract dominance-based interval-valued intuitionistic fuzzy rules [11]. Hu proposed generalized interval-valued fuzzy variable precision rough sets, which considers interval-valued fuzzy sets and variable precision simultaneously [51]. In order to handle highly uncertain data set, Zhao et al introduced the concept of variable precision interval type-2 fuzzy rough sets (VPI2FRS) by combining variable precision fuzzy rough sets and interval type-2 fuzzy rough sets [52].

With the era of big data coming, however, the volume of information is constantly increasing at an unprecedented rate. And the attribute set of an information system always varies over time with the collection of new information, which leads to the dynamic change of the rough approximations in RST. One possible approach to deal with such dynamic data is to restart the corresponding algorithms completely every time the new data arrive. However, this is obviously extremely inefficient, especially in the case of big data. Therefore, it is necessary to develop the techniques to update related knowledge effectively. Incremental learning approaches, which take full advantages of previous results instead of recalculate the whole data set, have been adopted in RST-based data analysis and decision making in many different kinds of information systems. Li et al. proposed incremental approaches for updating rough approximations under the characteristic relation-based rough sets for dynamic attribute generalization [15]. Luo et al. investigated the incremental strategies for maintaining rough approximations in dynamic set-valued decision systems [18-20]. Li et al. proposed incremental approaches for updating approximations based on dominance-based rough set model when some attributes are added into or deleted from an ordered information system [16]. Zeng et al. proposed the fuzzy information granulation methods to incrementally update approximations in hybrid information systems under the variation of the attribute set [43]. Chen et al. proposed incremental approaches for dynamic maintenance of rough approximations w.r.t. the addition of objects and attributes simultaneously based on decision-theoretic rough sets [6]. Zhang et al. established a composite rough set model to process attributes of multiple different types simultaneously in composite information systems and then proposed a novel matrix-based method for fast updating approximations in dynamic composite information systems [46, 47]. Since the IvIS is an important form of information representation in the real-world application, in this paper we mainly focus on the incremental mechanisms for updating rough approximations when an attribute set is added into or deleted from the IvIS.

In Section 2, we first review some basic concepts and similarity-based RST in IvIS. In Section 3, we represent the inclusion relationships between the original rough approximations and the updated ones after the attribute set changes by the Venn diagram for the first time, and then propose the incremental principles for updating rough approximations 
under the attribute generalization in the IvIS. Then the corresponding incremental algorithms for computing rough approximations are designed in Section 4. Furthermore, evaluation experiments on data sets from UCI as well as user-defined data sets are presented in Section 5. Finally, we conclude this paper with a brief summary and future work in Section 6.

\section{Preliminaries}

In this section, some basic concepts of IvIS and RST are reviewed [5]. First, we review some basic concepts about interval [22].

A closed interval on the real number set $\mathbb{R}$, denoted by $I=\left[b^{-}, b^{+}\right]$, is a set of real numbers given by $\left[b^{-}, b^{+}\right]=$ $\left\{x \in \mathbb{R} \mid b^{-} \leqslant x \leqslant b^{+}\right\}$, where $b^{-}$and $b^{+}$are called the lower and upper bounds of $I$, respectively. If $b^{-}=b^{+}$, the interval $I$ will degenerate into a single real number.

Suppose $I_{1}=\left[b_{1}^{-}, b_{1}^{+}\right]$and $I_{2}=\left[b_{2}^{-}, b_{2}^{+}\right]$are two intervals.

The intersection $I_{1} \cap I_{2}$ is defined as an interval

$$
I_{1} \cap I_{2}=\left\{\begin{array}{cc}
{\left[\max \left\{b_{1}^{-}, b_{2}^{-}\right\}, \min \left\{b_{1}^{+}, b_{2}^{+}\right\}\right],} & \max \left\{b_{1}^{-}, b_{2}^{-}\right\} \leq \min \left\{b_{1}^{+}, b_{2}^{+}\right\} \\
\emptyset, & \text { otherwise }
\end{array}\right.
$$

The union $I_{1} \cup I_{2}$ is also defined as an interval

$$
I_{1} \cup I_{2}=\left[\min \left\{b_{1}^{-}, b_{2}^{-}\right\}, \max \left\{b_{1}^{+}, b_{2}^{+}\right\}\right]
$$

From Eqs. (1) and (2), it is easy to obtain that $I_{1} \cap I_{2} \subseteq I_{1} \cup I_{2}$.

Definition 1. [5] An Interval-valued Information System (IvIS) is a quadruple ( $U, A T, V, f)$, where $U=\left\{x_{1}, x_{2}, \ldots, x_{n}\right\}$ is a non-empty finite set of objects called the universe, $A T=\left\{a_{1}, a_{2}, \ldots, a_{l}\right\}$ is a non-empty finite set of attributes, $V=\bigcup_{a \in A T} V_{a}$ and $V_{a}$ is called the domain of attribute $a$, and $f: U \times A T \rightarrow V$ is called a total function, such that $f(x, a)=\left[a(x)^{-}, a(x)^{+}\right] \in V_{a}$ is an interval for every $a \in A T, x \in U$, where $V_{a}$ is a set of intervals.

Table 1: An interval-valued information system.

\begin{tabular}{lccccc}
\hline & $a_{1}$ & $a_{2}$ & $a_{3}$ & $a_{4}$ & $a_{5}$ \\
\hline$x_{1}$ & {$[0.1,0.6]$} & {$[0.4,0.6]$} & {$[0.2,0.4]$} & {$[0.0,0.7]$} & {$[0.2,0.4]$} \\
$x_{2}$ & {$[0.6,0.8]$} & {$[0.4,0.6]$} & {$[0.3,0.5]$} & {$[0.8,1.0]$} & {$[0.5,0.8]$} \\
$x_{3}$ & {$[0.2,0.4]$} & {$[0.0,0.4]$} & {$[0.2,0.8]$} & {$[0.6,0.7]$} & {$[0.1,0.5]$} \\
$x_{4}$ & {$[0.7,0.8]$} & {$[0.2,0.6]$} & {$[0.3,0.6]$} & {$[0.2,0.5]$} & {$[0.4,0.9]$} \\
$x_{5}$ & {$[0.3,0.4]$} & {$[0.2,0.3]$} & {$[0.4,0.8]$} & {$[0.4,0.6]$} & {$[0.4,1.0]$} \\
$x_{6}$ & {$[0.4,0.9]$} & {$[0.1,0.8]$} & {$[0.2,0.8]$} & {$[0.1,0.6]$} & {$[0.5,0.7]$} \\
$x_{7}$ & {$[0.6,0.7]$} & {$[0.3,0.7]$} & {$[0.1,0.2]$} & {$[0.8,0.8]$} & {$[0.2,0.6]$} \\
$x_{8}$ & {$[0.6,1.0]$} & {$[0.7,0.9]$} & {$[0.2,0.4]$} & {$[0.4,0.8]$} & {$[0.4,0.8]$} \\
$x_{9}$ & {$[0.1,0.2]$} & {$[0.1,0.1]$} & {$[0.3,0.6]$} & {$[0.7,0.9]$} & {$[0.3,0.5]$} \\
\hline
\end{tabular}

Definition 2. [5] Let $(U, A T, V, f)$ be an IvIS, $x_{i}, x_{j} \in U$, and $a_{k} \in A T$. The similarity degree between $x_{i}$ and $x_{j}$ under the attribute $a_{k}$ is defined as follows:

$$
S_{i j}^{k}=\frac{\left|f\left(x_{i}, a_{k}\right) \cap f\left(x_{j}, a_{k}\right)\right|}{\left|f\left(x_{i}, a_{k}\right) \cup f\left(x_{j}, a_{k}\right)\right|}
$$

where $i, j=1,2, \ldots, n, k=1,2, \ldots, m,|\cdot|$ denotes the length of closed interval, and the length of an empty interval or a single point is zero. 
Obviously, the similarity degree $S_{i j}^{k}$ satisfies the following properties:

1. $0 \leq S_{i j}^{k} \leq 1$;

2. $S_{i j}^{k}=0$ if and only if $f\left(x_{i}, a_{k}\right) \cap f\left(x_{j}, a_{k}\right)$ is empty or single value;

3. $S_{i j}^{k}=1$ if and only if $f\left(x_{i}, a_{k}\right)=f\left(x_{j}, a_{k}\right)$;

4. $S_{i j}^{k}=S_{j i}^{k}$.

Definition 3. [5] Let $(U, A T, V, f)$ be an $I v I S, x_{i}, x_{j} \in U, A \subseteq A T$ and $\lambda \in[0.5,1]$. The $\lambda$-similarity relation with respect to $A$ is defined as follows:

$$
S_{A}^{\lambda}=\left\{\left(x_{i}, x_{j}\right) \in U \times U \mid S_{i j}^{k} \geq \lambda, \forall a_{k} \in A\right\}
$$

where $S_{i j}^{k}$ is the similarity degree between $x_{i}$ and $x_{j}, \lambda$ is called the similarity degree threshold.

Obviously, $S_{A}^{\lambda}$ is reflexive and symmetric, but not transitive. It is also called the tolerance relation which satisfies $S_{A}^{\lambda}=\bigcap_{a \in A} S_{a}^{\lambda}$. And the $\lambda$-similarity class containing $x_{i}$ is defined as $S_{A}^{\lambda}\left(x_{i}\right)=\left\{x_{j} \in U \mid\left(x_{i}, x_{j}\right) \in S_{A}^{\lambda}\right\}$.

Lemma 1. Let $(U, A T, V, f)$ be an IvIS. $A_{1}, A_{2}$ are any two subsets of the attribute set $A T . \forall x \in U$, we have: (1) $S_{A_{1} \cup A_{2}}^{\lambda}(x) \subseteq S_{A_{1}}^{\lambda}(x) ; \quad$ (2) $S_{A_{1} \cup A_{2}}^{\lambda}(x) \subseteq S_{A_{2}}^{\lambda}(x)$.

Definition 4. [5] Let $(U, A T, V, f)$ be an IvIS. $A \subseteq A T, \lambda \in[0.5,1]$, and $\forall X \in U$, the lower and upper approximations of $X$ are defined respectively as:

$$
\underline{A}^{\lambda}(X)=\left\{x_{i} \in U \mid S_{A}^{\lambda}\left(x_{i}\right) \subseteq X\right\}, \quad \bar{A}^{\lambda}(X)=\left\{x_{i} \in U \mid\left(S_{A}^{\lambda}\left(x_{i}\right) \cap X\right) \neq \emptyset\right\} .
$$

\section{Incremental Approaches for Updating Rough Approximations under the Attribute Generalization}

In this section, we propose the incremental approaches for updating rough approximations in the view of $\lambda$ similarity-based rough set approach when an attribute set is added into or removed from the IvIS, respectively [48].

Proposition 1. Let $(U, A T, V, f)$ be an $I v I S, \lambda \in[0.5,1], A_{1}, A_{2} \subseteq A T . \forall X \subseteq U$, we have: (1) $A_{1}^{\lambda}(X) \cup A_{2}^{\lambda}(X) \subseteq A_{1} \cup A_{2}^{\lambda}(X)$;

(2) ${\overline{\overline{A_{1}}}}^{\lambda}(X) \cap{\overline{\overline{A_{2}}}}^{\lambda}(X) \supseteq{\overline{\overline{A_{1} \cup A_{2}}}}^{\lambda}(X)$.

Proof. (1) $\forall x \in \underline{A_{1}}{ }^{\lambda}(X) \cup \underline{A_{2}}{ }^{\lambda}(X), x \in \underline{A_{1}}{ }^{\lambda}(X) \vee x \in{\underline{A_{2}}}^{\lambda}(X)$, namely, $S_{A_{1}}^{\lambda}(x) \subseteq X \vee S_{A_{2}}^{\lambda}(x) \subseteq X$. According to Lemma 1, we can get $S_{A_{1} \cup A_{2}}^{\lambda}(x) \subseteq \bar{X}$, so $x \in \underline{A_{1} \cup A_{2}^{\lambda}}(X)$. Finally, we have $\underline{A_{1}}{ }^{\lambda}(X) \cup \underline{A_{2}}{ }^{\lambda}(X) \subseteq \underline{A_{1} \cup A_{2}}{ }^{\lambda}(X)$.

(2) $\forall x \in{\overline{A_{1} \cup A_{2}}}^{\lambda}(X), S_{A_{1} \cup A_{2}}^{\lambda}(x) \cap X \neq \emptyset$. According to Lemma 1 , we can have $S_{A_{1}}^{\lambda}(x) \cap X \neq \emptyset \wedge S_{A_{2}}^{\lambda}(x) \cap X \neq \emptyset$. Then $x \in{\overline{A_{1}}}^{\lambda}(X) \wedge x \in{\overline{A_{2}}}^{\lambda}(X)$, that is, $x \in{\overline{A_{1}}}^{\lambda}(X) \cap{\overline{A_{2}}}^{\lambda}(X)$. Finally, ${\overline{A_{1}}}^{\lambda}(X) \cap{\overline{A_{2}}}^{\lambda}(X) \supseteq{\overline{A_{1} \cup A_{2}}}^{\lambda}(X)$.

Example 1. In the IvIS given by Table 1, let $X=\left\{x_{1}, x_{4}, x_{6}\right\}$. If $\lambda=0.5, A_{1}=\left\{a_{1}, a_{2}, a_{5}\right\}, A_{2}=\left\{a_{2}, a_{3}\right\}$, we can obtain the lower and upper approximations of $X$ w.r.t. $A_{1}$ and $A_{2}$ as follows:

For $A_{1}$, we have

$$
\begin{aligned}
& S_{A_{1}}^{0.5}\left(x_{1}\right)=\left\{x_{1}\right\}, \quad S_{A_{1}}^{0.5}\left(x_{2}\right)=\left\{x_{2}, x_{4}\right\}, \quad S_{A_{1}}^{0.5}\left(x_{3}\right)=\left\{x_{3}\right\}, \quad S_{A_{1}}^{0.5}\left(x_{4}\right)=\left\{x_{2}, x_{4}\right\}, \quad S_{A_{1}}^{0.5}\left(x_{5}\right)=\left\{x_{5}\right\}, \\
& S_{A_{1}}^{0.5}\left(x_{6}\right)=\left\{x_{6}\right\}, \quad S_{A_{1}}^{0.5}\left(x_{7}\right)=\left\{x_{7}\right\}, \quad S_{A_{1}}^{0.5}\left(x_{8}\right)=\left\{x_{8}\right\}, \quad S_{A_{1}}^{0.5}\left(x_{9}\right)=\left\{x_{9}\right\} .
\end{aligned}
$$

and

$A_{1}^{0.5}(X)=\left\{x_{1}, x_{6}\right\}, \quad{\overline{A_{1}}}^{0.5}(X)=\left\{x_{1}, x_{2}, x_{4}, x_{6}\right\}$.

For $\overline{A_{2}}$, we have

$S_{A_{2}}^{0.5}\left(x_{1}\right)=\left\{x_{1}\right\}, \quad S_{A_{2}}^{0.5}\left(x_{2}\right)=\left\{x_{2}, x_{4}\right\}, \quad S_{A_{2}}^{0.5}\left(x_{3}\right)=\left\{x_{3}\right\}, \quad S_{A_{2}}^{0.5}\left(x_{4}\right)=\left\{x_{2}, x_{4}, x_{6}\right\}, \quad S_{A_{2}}^{0.5}\left(x_{5}\right)=\left\{x_{5}\right\}$, and$$
S_{A_{2}}^{0.5}\left(x_{6}\right)=\left\{x_{4}, x_{6}\right\}, \quad S_{A_{2}}^{0.5}\left(x_{7}\right)=\left\{x_{7}\right\}, \quad S_{A_{2}}^{0.5}\left(x_{8}\right)=\left\{x_{8}\right\}, \quad S_{A_{2}}^{0.5}\left(x_{9}\right)=\left\{x_{9}\right\} .
$$ 


$$
\begin{aligned}
& {\underline{A_{2}}}^{0.5}(X)=\left\{x_{1}, x_{6}\right\}, \quad{\overline{A_{2}}}^{0.5}(X)=\left\{x_{1}, x_{2}, x_{4}, x_{6}\right\} . \\
& \text { For } \overline{A_{1}} \cup A_{2} \text {, we have } \\
& S_{A_{1} \cup A_{2}}^{0.5}\left(x_{1}\right)=\left\{x_{1}\right\}, \quad S_{A_{1} \cup A_{2}}^{0.5}\left(x_{2}\right)=\left\{x_{2}, x_{4}\right\}, \quad S_{A_{1} \cup A_{2}}^{0.5}\left(x_{3}\right)=\left\{x_{3}\right\}, \quad S_{A_{1} \cup A_{2}}^{0.5}\left(x_{4}\right)=\left\{x_{2}, x_{4}\right\}, \quad S_{A_{1} \cup A_{2}}^{0.5}\left(x_{5}\right)=\left\{x_{5}\right\}, \\
& S_{A_{1} \cup A_{2}}^{0.5}\left(x_{6}\right)=\left\{x_{6}\right\}, \quad S_{A_{1} \cup A_{2}}^{0.5}\left(x_{7}\right)=\left\{x_{7}\right\}, \quad S_{A_{1} \cup A_{2}}^{0.5}\left(x_{8}\right)=\left\{x_{8}\right\}, \quad S_{A_{1} \cup A_{2}}^{0.5}\left(x_{9}\right)=\left\{x_{9}\right\} . \\
& {\underline{A_{1} \cup A_{2}}}^{0.5}(X)=\left\{x_{1}, x_{6}\right\} \supseteq{\underline{A_{1}}}^{0.5}(X) \cup{\underline{A_{2}}}^{0.5}(X), \quad{\overline{A_{1} \cup A_{2}}}^{0.5}(X)=\left\{x_{1}, x_{2}, x_{4}, x_{6}\right\} \subseteq{\overline{A_{1}}}^{0.5}(X) \cap{\overline{A_{2}}}^{0.5}(X) .
\end{aligned}
$$

Proposition 2. Let $(U, A T, V, f)$ be an IvIS, $\lambda \in[0.5,1], A_{1}, A_{2} \subseteq A T$ and $A_{2} \subseteq A_{1} . \forall X \subseteq U$, we have: (1) $\underline{A}_{1}-A_{2}^{\lambda}(X) \subseteq \underline{A_{1}^{\lambda}}(X)$

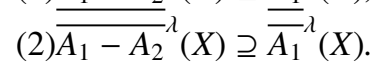

Proof. (1) $\forall x \in \underline{A_{1}-A_{2}}{ }^{\lambda}(X), S_{A_{1}-A_{2}}^{\lambda}(x) \subseteq X$. According to Lemma 1, we know that $S_{A_{1}}^{\lambda}(x) \subseteq S_{A_{1}-A_{2}}^{\lambda}(x)$, so we have $S_{A_{1}}^{\lambda}(x) \subseteq X$, namely, $x \in \underline{A_{1}}{ }^{\lambda}(X)$. Finally, we have $\underline{A_{1}-A_{2}}{ }^{\lambda}(X) \subseteq \underline{A_{1}}{ }^{\lambda}(X)$.

(2) $\forall x \in{\overline{A_{1}}}^{\lambda}(X), S_{A_{1}}^{\lambda}(x) \overline{\cap X} \neq \emptyset$. Similar to (1), we have $S_{A_{1}-A_{2}}^{\lambda} \overline{(x)} \cap X \neq \emptyset$, namely, $x \in \overline{A_{1}-A_{2}}(X)$. Finally, we have ${\overline{A_{1}-A_{2}}}^{\lambda}(X) \supseteq{\overline{A_{1}}}^{\lambda}(X)$.

Example 2. (Continued from Example 1) In the IvIS given by Table 1, let $X=\left\{x_{1}, x_{4}, x_{6}\right\}$. If $\lambda=0.5$, $A_{1}=$ $\left\{a_{1}, a_{2}, a_{3}, a_{5}\right\}, A_{2}=\left\{a_{1}, a_{5}\right\}$, from Example 1 we have

$$
\begin{aligned}
& \quad{\underline{A_{1}}}^{0.5}(X)=\left\{x_{1}, x_{6}\right\}, \quad{\overline{A_{1}}}^{0.5}(X)=\left\{x_{1}, x_{2}, x_{4}, x_{6}\right\} . \\
& \text { and } \\
& \quad{\underline{A_{1}-A_{2}}}^{0.5}(X)=\left\{x_{1}, x_{6}\right\} \subseteq \underline{A 1}^{0.5}(X), \quad{\overline{A_{1}-A_{2}}}^{0.5}(X)=\left\{x_{1}, x_{2}, x_{4}, x_{6}\right\} \supseteq{\overline{A_{1}}}^{0.5}(X) .
\end{aligned}
$$

Propositions 1 and 2 show that the lower approximation becomes bigger and the upper approximation becomes smaller when the attribute set enlarges and the lower approximation becomes smaller and the upper approximation becomes bigger when the attribute set narrows. Based on the principles, we can obtain the incremental approaches for updating rough approximations when adding an attribute set or deleting an attribute set in IvIS as follows.

\subsection{Adding a New Attribute Set}

Let $(U, A T, V, f)$ be the original IvIS, and $\left(U, A^{+}, V^{+}, f^{+}\right)$denote the IvIS which is formed by the newly added attributes. And $\forall X \subseteq U$, the updated lower and upper approximations of $X$ are denoted as $\underline{A T \cup A^{+\lambda}(X) \text { and }}$ ${\overline{A T \cup A^{+}}}^{\lambda}(X)$, respectively. Considering the addition of an attribute set $A^{+}$in the IvIS, the following incremental mechanisms are proposed for updating rough approximations.

Proposition 3. Let $(U, A T, V, f)$ be the IvIS. $\forall X \subseteq U$, the lower approximation of $X$ with the addition of $A^{+}$can be updated as follows:

$$
\underline{A T \cup A^{+\lambda}}(X)=\underline{A T^{\lambda}}(X) \cup \underline{A^{+\lambda}}(X) \cup Y_{1}
$$

where $Y_{1}=\left\{x \in\left(X-\underline{A T^{\lambda}}(X)\right) \cap\left(X-\underline{A^{+\lambda}}(X)\right) \mid S_{A T \cup A^{+}}^{\lambda}(x) \subseteq X\right\}$.

Proof. According to Proposition 1 and the definition of lower approximation, we have $\underline{A T^{\lambda}}(X) \cup \underline{A^{+\lambda}}(X) \subseteq \underline{A T \cup A^{+\lambda}}(X) \subseteq$

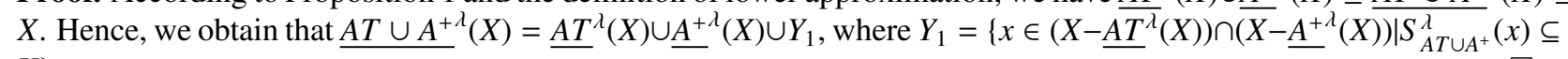
$X\}$.

Example 3. (Continued from Example 2) Given the IvIS $(U, A T, V, f)$ as shown in Table 1 , the attribute set $A^{+}=$ $\left\{a_{6}, a_{7}\right\}$ shown in Table 2 is added into Table 1: 
Table 2: The attribute set $A^{+}=\left\{a_{6}, a_{7}\right\}$ is inserted into Table 1 .

\begin{tabular}{lcc}
\hline & $a_{6}$ & $a_{7}$ \\
\hline$x_{1}$ & {$[0.1,0.4]$} & {$[0.3,0.5]$} \\
$x_{2}$ & {$[0.2,0.5]$} & {$[0.4,0.7]$} \\
$x_{3}$ & {$[0.4,0.7]$} & {$[0.1,0.5]$} \\
$x_{4}$ & {$[0.6,0.7]$} & {$[0.1,0.4]$} \\
$x_{5}$ & {$[0.3,0.4]$} & {$[0.2,0.6]$} \\
$x_{6}$ & {$[0.5,0.8]$} & {$[0.5,0.8]$} \\
$x_{7}$ & {$[0.1,0.3]$} & {$[0.4,0.5]$} \\
$x_{8}$ & {$[0.2,0.4]$} & {$[0.3,0.7]$} \\
$x_{9}$ & {$[0.7,0.9]$} & {$[0.2,0.5]$} \\
\hline
\end{tabular}

In the following, we suppose that $X=\left\{x_{1}, x_{4}, x_{6}\right\}$ similar to Example 1. From Table 1, we have

$S_{A T}^{0.5}\left(x_{1}\right)=\left\{x_{1}\right\}, \quad S_{A T}^{0.5}\left(x_{2}\right)=\left\{x_{2}\right\}, \quad S_{A T}^{0.5}\left(x_{3}\right)=\left\{x_{3}\right\}, \quad S_{A T}^{0.5}\left(x_{4}\right)=\left\{x_{4}\right\}, \quad S_{A T}^{0.5}\left(x_{5}\right)=\left\{x_{5}\right\}$, $S_{A T}^{0.5}\left(x_{6}\right)=\left\{x_{6}\right\}, \quad S_{A T}^{0.5}\left(x_{7}\right)=\left\{x_{7}\right\}, \quad S_{A T}^{0.5}\left(x_{8}\right)=\left\{x_{8}\right\}, \quad S_{A T}^{0.5}\left(x_{9}\right)=\left\{x_{9}\right\}$.

Then according to Definition 4, we have $\underline{A T}^{0.5}(X)=\left\{x_{1}, x_{4}, x_{6}\right\}$ and $\overline{A T}^{0.5}(X)=\left\{x_{1}, x_{4}, x_{6}\right\}$.

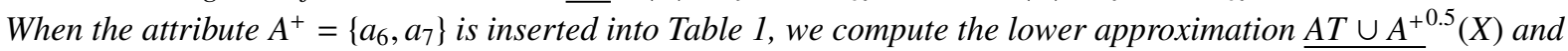
the upper approximation $\overline{A T \cup A^{+}} .5$ (X) by the non-incremental methods as follows:

First we obtain the 0.5-similarity classes about $A T \cup A^{+}$:

$S_{A T \cup A^{+}}^{0.5}\left(x_{1}\right)=\left\{x_{1}\right\}, \quad S_{A T \cup A^{+}}^{0.5}\left(x_{2}\right)=\left\{x_{2}\right\}, \quad S_{A T \cup A^{+}}^{0.5}\left(x_{3}\right)=\left\{x_{3}\right\}, \quad S_{A T \cup A^{+}}^{0.5}\left(x_{4}\right)=\left\{x_{4}\right\}, \quad S_{A T \cup A^{+}}^{0.5}\left(x_{5}\right)=\left\{x_{5}\right\}$,

$S_{A T \cup A^{+}}^{0.5}\left(x_{6}\right)=\left\{x_{6}\right\}, \quad S_{A T \cup A^{+}}^{0.5}\left(x_{7}\right)=\left\{x_{7}\right\}, \quad S_{A T \cup A^{+}}^{0.5}\left(x_{8}\right)=\left\{x_{8}\right\}, \quad S_{A T \cup A^{+}}^{0.5}\left(x_{9}\right)=\left\{x_{9}\right\}$.

Then according to Definition 4, we have $\underline{A T \cup A^{+0.5}}(X)=\left\{x_{1}, x_{4}, x_{6}\right\}$ and $\overline{A T \cup A^{+}}{ }^{0.5}(X)=\left\{x_{1}, x_{4}, x_{6}\right\}$.

We update the lower approximation by Proposition 3 as follows:

From Table 2, we have

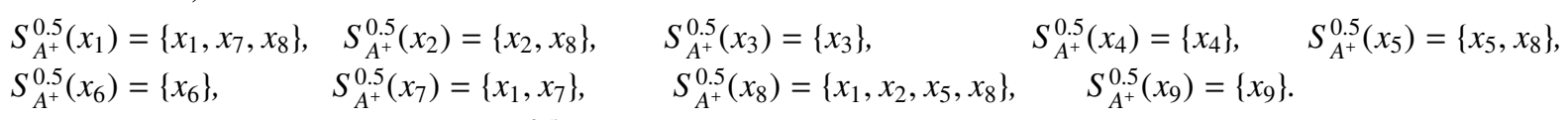

So we have $\underline{A}^{+0.5}(X)=\left\{x_{4}, x_{6}\right\}$ and ${\overline{A^{+}}}^{0.5}(X)=\left\{x_{1}, x_{4}, x_{6}, x_{7}, x_{8}\right\}$. Then we have $\left(X-\underline{A T}^{0.5}(X)\right) \cap\left(X-\underline{A}^{+0.5}(X)\right)=\emptyset$, so $Y_{1}=\emptyset$. Thus $\underline{A T \cup A^{+0.5}}(X)=\underline{A T^{0.5}}(X) \cup \underline{A^{+0.5}}(X) \cup Y_{1}=\left\{x_{1}, x_{4}, x_{6}\right\}$, which is the same as the result of nonincremental methods.

Proposition 4. Let $(U, A T, V, f)$ be the IvIS. $\forall X \subseteq U$, the upper approximation of $X$ with the addition of $A^{+}$can be updated as follows:

$$
{\overline{A T \cup A^{+}}}^{\lambda}(X)=\overline{A T}^{\lambda}(X) \cap{\overline{A^{+}}}^{\lambda}(X) \backslash Z_{1}
$$

where $Z_{1}=\left\{x \in\left(\overline{A T}^{\lambda}(X) \cap{\overline{A^{+}}}^{\lambda}(X)-X\right) \mid S_{A T \cup A^{+}}^{\lambda}(x) \cap X=\emptyset\right\}$.

Proof. According to Proposition 1 and the definition of upper approximation, we have $X \subseteq{\overline{A T \cup A^{+}}}^{\lambda}(X) \subseteq \overline{A T}^{\lambda}(X) \cap$ ${\overline{A^{+}}}^{\lambda}(X)$. Therefore, we obtain that ${\overline{A T \cup A^{+}}}^{\lambda}(X)=\overline{A T}^{\lambda}(X) \cap{\overline{A^{+}}}^{\lambda}(X) \backslash Z_{1}$, where $Z_{1}=\left\{x \in\left(\overline{A T}^{\lambda}(X) \cap{\overline{A^{+}}}^{\lambda}(X)-\right.\right.$ $\left.X) \mid S_{A T \cup A^{+}}^{\lambda}(x) \cap X=\emptyset\right\}$.

Example 4. (Continued from Example 3) When the attribute $A^{+}=\left\{a_{6}, a_{7}\right\}$ is inserted into Table 1, we update the upper approximation by Proposition 4 as follows:

Firstly, we have $\overline{A T}^{0.5}(X) \cap{\overline{A^{+}}}^{0.5}(X)-X=\emptyset$, so $Z_{1}=\emptyset$. Thus ${\overline{A T \cup A^{+}}}^{0.5}(X)=\overline{A T}^{0.5}(X) \cap{\overline{A^{+}}}^{0.5}(X) \backslash Z_{1}=$ $\left\{x_{1}, x_{4}, x_{6}\right\}$, which is the same as the result of non-incremental methods. 


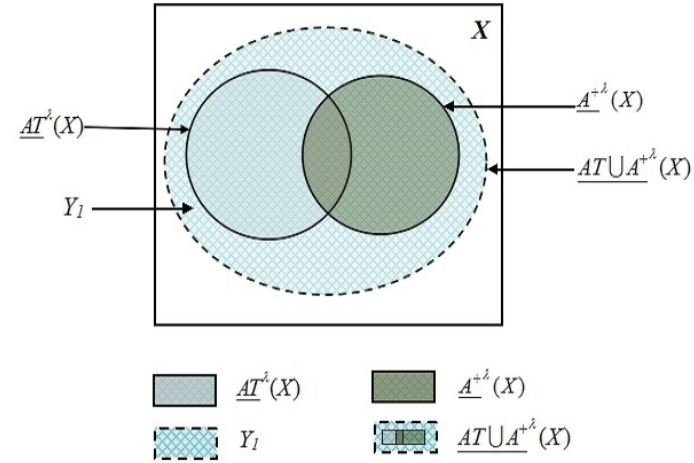

(a) The mechanism of incremental updating lower approximation

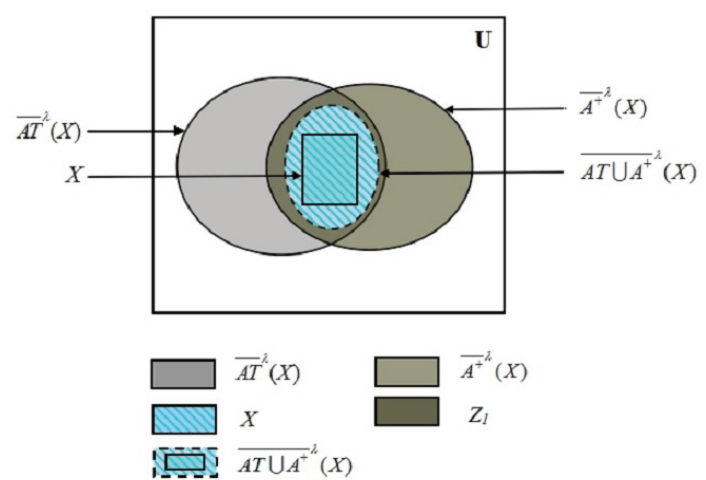

(b) The mechanism of incremental updating upper approximation

Figure 1: The mechanisms of incremental updating approximations when adding an attribute set

Fig. 1(a) and Fig. 1(b) present the incremental mechanisms for updating lower and upper approximations when adding an attribute set $A^{+}$, respectively. The dotted circles in Fig. 1 denote the updated lower and upper approximations. From Fig. 1(a), it's clear that $\left(A^{+\lambda}(X) \backslash\left(A^{+\lambda}(X) \cap A T^{\lambda}(X)\right)\right) \cup Y_{1}$ is the incremental part of the updated lower approximation with respect to the original lower approximation $\underline{A T^{\lambda}}(X)$. From Fig. 1(b), we know that $Z_{1}$ is the incremental part of the updated upper approximation.

\subsection{Deleting an Attribute Set}

Let $(U, A T, V, f)$ be the original IvIS, $\left(U, A^{-}, V, f\right)$ denote the IvIS which is formed by the deleted attributes, and $\left(U, A T^{\prime}, V, f\right)$ denote the remaining IvIS after deleting the attribute set $A^{-}$. Then $\forall X \subseteq U$, the updated lower and upper approximations of $X$ are defined as $\underline{A T^{\prime \lambda}}(X)$ and ${\overline{A T^{\prime}}}^{\lambda}(X)$, respectively. Considering the deletion of an attribute set $A^{-}$ from the IvIS, the following propositions are proposed for updating rough approximations, where $A T^{\prime}$ is defined as the updated attribute set, i.e., $A T^{\prime}=A T-A^{-}$.

Proposition 5. Let $(U, A T, V, f)$ be the IvIS. $\forall X \subseteq U$, the lower approximation of $X$ with the deletion of $A^{-}$can be updated as follows:

$$
{\underline{A T^{\prime}}}^{\lambda}(X)=\underline{A T^{\lambda}}(X) \backslash Y_{2}
$$

where $Y_{2}=\left\{x \in \underline{A T^{\lambda}}(X) \mid S_{A T^{\prime}}^{\lambda}(x) \nsubseteq X\right\}$.

Proof. According to Proposition 2 and the definition of lower approximation, we have $\underline{A T^{\prime \lambda}}(X) \subseteq \underline{A T^{\lambda}}(X)$. Then we can obtain $\underline{A T^{\prime \lambda}}(X)=\underline{A T^{\lambda}}(X)-Y_{2}$, where $Y_{2}=\left\{x \in \underline{A T^{\lambda}}(X) \mid S_{A T^{\prime}}^{\lambda}(x) \nsubseteq X\right\}$.

Example 5. Given the IvIS $(U, A T, V, f)$ as shown in Table 1 , the attribute set $A^{-}=\left\{a_{3}, a_{4}\right\}$ is removed from Table 1 . When the attribute $A^{-}=\left\{a_{3}, a_{4}\right\}$ is deleted from Table 1, we calculate the updated lower approximation by Proposition 5 as follows:

First, we know $A T^{\prime}=A=\left\{a_{1}, a_{2}, a_{5}\right\}$ and the results of $S_{A T^{\prime}}^{0.5}\left(x_{i}\right)$ for $i=1,2, \ldots, 9$ from Example 1.

Then according to Propositions 5, if $X=\left\{x_{1}, x_{4}, x_{6}\right\}$, we have $Y_{2}=\left\{x \in \underline{A T}^{0.5}(X) \mid S_{A T^{\prime}}^{0.5}(x) \nsubseteq X\right\}=\left\{x_{4}\right\}$.

Therefore, $\underline{A T^{\prime 0.5}}(X)=\underline{A T^{0.5}}(X) \backslash Y_{2}=\left\{x_{1}, x_{6}\right\}$, which is the same as the result of the non-incremental result in Example 1.

Proposition 6. Let $(U, A T, V, f)$ be the IvIS. $\forall X \subseteq U$, the upper approximation of $X$ with the deletion of $A^{-}$can be updated as follows:

$$
{\overline{A T^{\prime}}}^{\lambda}(X)=\overline{A T}^{\lambda}(X) \cup Z_{2}
$$

where $Z_{2}=\left\{x \in\left(U-\overline{A T}^{\lambda}(X)\right) \mid S_{A T^{\prime}}^{\lambda}(x) \cap X \neq \emptyset\right\}$. 
Proof. According to Proposition 2 and the definition of upper approximation, we have that $\overline{A T}^{\lambda}(X) \subseteq{\overline{A T^{\prime}}}^{\lambda}(X)$. So we can get ${\overline{A T^{\prime}}}^{\lambda}(X)=\overline{A T}^{\lambda}(X) \cup Z_{2}$, where $Z_{2}=\left\{x \in\left(U-\overline{A T}^{\lambda}(X)\right) \mid S_{A T^{\prime}}^{\lambda}(x) \cap X \neq \emptyset\right\}$.

Example 6. (Continued from Example 5) When the attribute $A^{-}=\left\{a_{3}, a_{4}\right\}$ is deleted from Table 1, we calculate the updated upper approximation by Proposition 6 as follows:

First, we have $Z_{2}=\left\{x \in\left(U-\overline{A T}^{0.5}(X)\right) \mid S_{A T^{\prime}}^{0.5}(x) \cap X \neq \emptyset\right\}=\left\{x_{2}\right\}$.

Therefore, ${\overline{A T^{\prime}}}^{0.5}(X)=\overline{A T}^{0.5}(X) \cup Z_{2}=\left\{x_{1}, x_{2}, x_{4}, x_{6}\right\}$, which is the same as the result of the non-incremental result in Example 1.

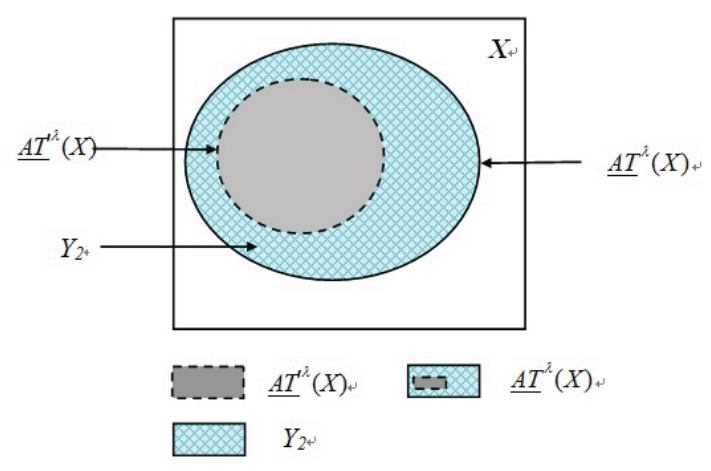

(a) The mechanism of incremental updating lower approximation

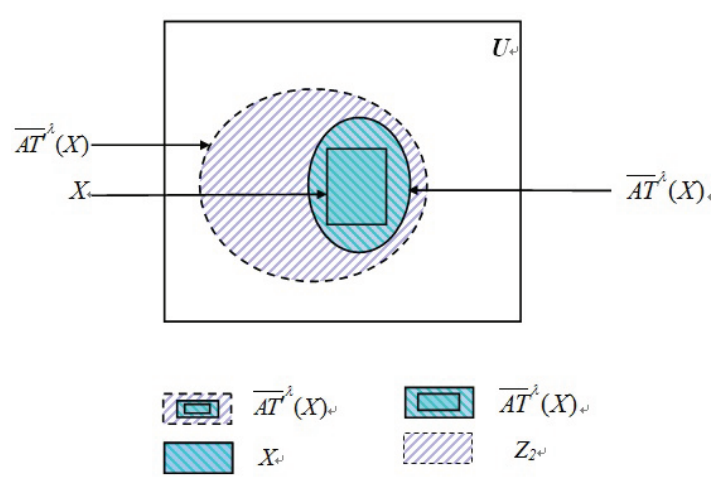

(b) The mechanism of incremental updating upper approximation

Figure 2: The mechanisms of incremental updating approximations when deleting an attribute set

Fig. 2(a) and Fig. 2(b) present the incremental mechanisms for updating lower and upper approximations when deleting an attribute set $A^{-}$, respectively. Similar to Fig. 1, the updated lower and upper approximations are characterized with dotted circles. In the figures, it is clear that $Y_{2}$ and $Z_{2}$ are the incremental parts of lower and upper approximations, respectively, with respect to the original rough approximations $\underline{A T}^{\lambda}(X)$ and $\overline{A T}^{\lambda}(X)$ when an attribute set is deleted from IvIS.

\section{Incremental Algorithms for Updating Rough Approximations in IvIS under Attribute Generalization}

In this section, we develop incremental algorithms for computing rough approximations on the variation of the attribute set in IvIS corresponding to the incremental mechanisms in Sections 2 and 3, respectively.

\subsection{The Static Algorithm of Computing Approximations in IvIS}

Algorithm 1 is a static (non-incremental) algorithm for computing the lower and upper approximations in IvIS. Steps 3-12 compute all the similarity classes of every object according to Definitions 1-3, whose time complexity is $O\left(|U|+|U|^{2}|A T|\right)=O\left(|U|^{2}|A T|\right)$. Steps 13-20 compute the lower approximation and upper approximation, respectively, whose time complexity is $O(|U|(|X|+|X|))=O(|U||X|)$, in which the time complexity of computing lower approximation and upper approximation are both $O(|U||X|)$. So the total time complexity of the static algorithm to compute lower approximation and upper approximation are both $O\left(|U|^{2}|A T|+|U||X|\right)$.

\subsection{The Incremental Algorithm of Updating Approximations when Adding an Attribute Set in IvIS}

Algorithm 2 is an incremental algorithm for updating the lower and upper approximations when adding an attribute set in IvIS. Step 4 computes all the similarity classes of every object and the approximations of $X$ with respect to the added attribute set $A^{+}$according to Algorithm 1, whose time complexity is $O\left(|U|^{2}\left|A^{+}\right|+|U \| X|\right)$. Steps 5-11 compute 


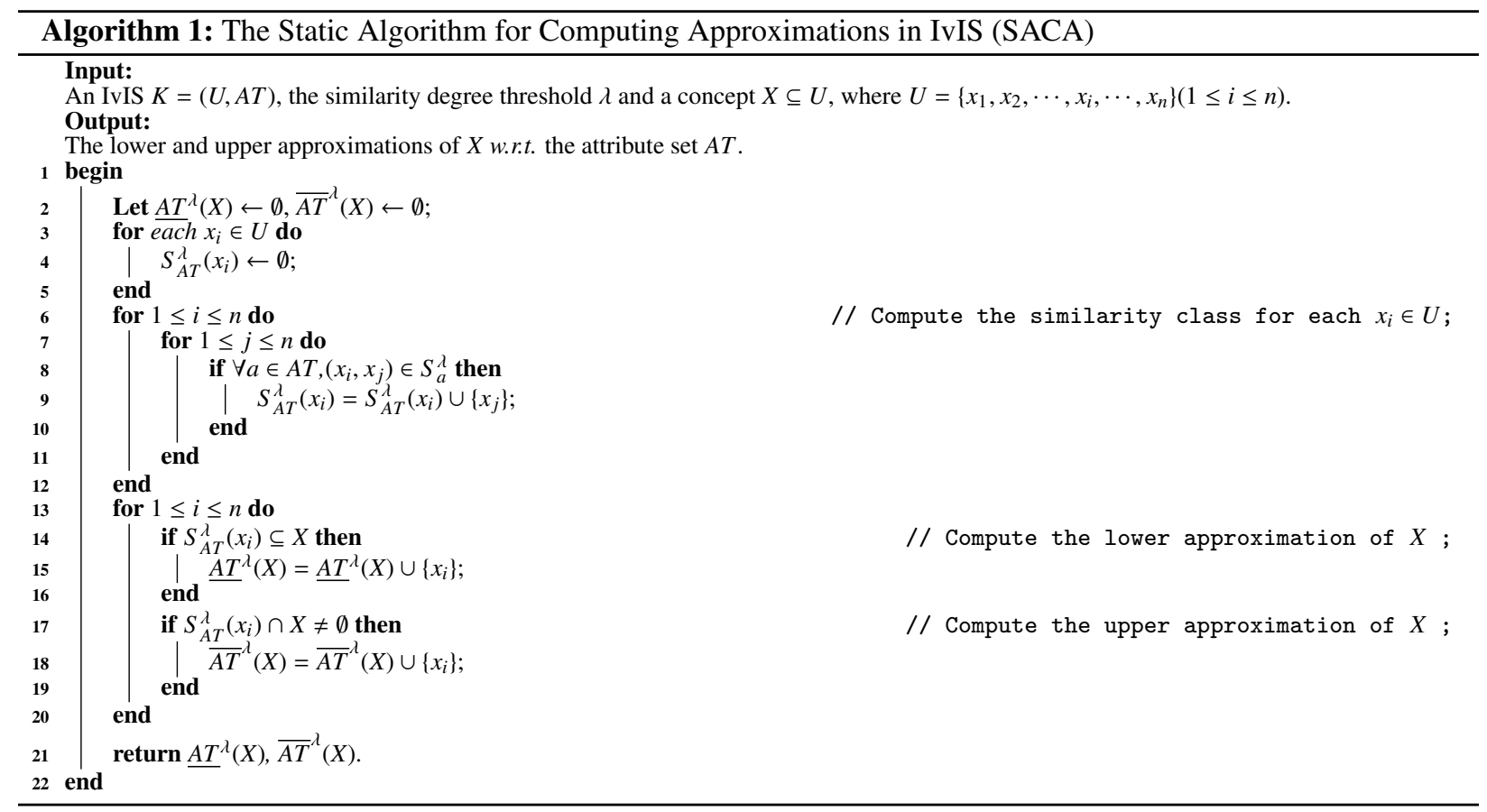

the incremental part $Y_{1}$ of the lower approximation by Proposition 3, whose time complexity is $O(|X|(|U|+|X|))=$ $O(|U||X|)$. Step 12 updates the lower approximation of $X$, whose time complexity is $O(|X|+|X|)=O(|X|)$. The total time complexity of updating the lower approximation is $O\left(|U|^{2}\left|A^{+}\right|+|U||X|+|X|\right)=O\left(|U|^{2}\left|A^{+}\right|+|U||X|\right)$. Steps 13-19 compute the incremental part $Z_{1}$ of the upper approximation by Proposition 4, whose time complexity is $O(|X|(|U|+|X|))=$ $O(|U||X|)$. Step 20 updates the upper approximation of $X$, whose time complexity is $O(|U|+|U|)=O(|U|)$. The total time complexity of updating the upper approximation is $O\left(|U|^{2}\left|A^{+}\right|+|U||X|+|U|\right)=O\left(|U|^{2}\left|A^{+}\right|+|U||X|\right)$. Clearly, it is lower than that of Algorithm 1.

\subsection{The Incremental Algorithm of Updating Approximations when Deleting an Attribute Set from IvIS}

Algorithm 3 is an incremental algorithm for updating the lower and upper approximations when deleting an attribute set from IvIS. Steps 4-13 compute the incremental part $Y_{2}$ of the lower approximation by Proposition 5, whose time complexity is $O\left(|X|\left(|U|\left|A T^{\prime}\right|+|X|\right)\right)=O\left(|U||X|\left|A T^{\prime}\right|+|X|^{2}\right)$. Step 14 updates the lower approximation of $X$, whose time complexity is $O\left(\left|A T^{\lambda}(X)\right|\right)$. The total time complexity of updating the lower approximation is $O\left(\left|U\|X\| A T^{\prime}\right|+\right.$ $\left.|X|^{2}+\underline{A T^{\lambda}}(X) \mid\right)=O\left(|\overline{U \|} X|\left|A T^{\prime}\right|+|X|^{2}\right)$, which is clearly less than that of Algorithm 1. Steps 15-25 compute the incremental part $Z_{2}$ of the upper approximation by Proposition 6, whose time complexity is $O\left(|U-X|\left(\left|U \| A T^{\prime}\right|+\right.\right.$ $|X|))=O\left(\left|U\left\|A T^{\prime}\right\| U-X\right|+|U-X||X|\right)$. Step 26 updates the upper approximation of $X$, whose time complexity is $O\left(\max \left\{\left|A T^{\lambda}(X)\right|,|U-X|\right\}\right)$. The total time complexity of updating the upper approximation is $O\left(\left|U \| A T^{\prime}\right||U-X|+\mid U-\right.$ $\left.X|| X \mid+\overline{\max }\left\{\left|A T^{\lambda}(X)\right|,|U-X|\right\}\right)=O\left(|U|\left|A T^{\prime}\right||U-X|+|U-X||X|\right)$, which is lower than that of Algorithm 1. However, it is almost as much as that of Algorithm 1 when the size of $X$ is very small.

\section{Experimental Evaluations}

To evaluate the performance of Algorithms 1-3, also named as Algorithms SACA, IAUA-A and IAUA-D, we download five data sets from the machine learning data repository, University of California at Irvine [3], in which the attribute values are all real numbers. Firstly, a preprocessing step which transforms the real-valued data sets to intervalvalued ones is conducted. This process commonly involves the discretization of continuous attribute values. Here we employ the statistical method in [14]. Let $(U, A T \cup\{d\}, V, f)$ be an real-valued decision system. $\forall a \in A T, x \in U$, we compute $a(x)^{-}=a(x)-2 \sigma_{d}, a(x)^{+}=a(x)+2 \sigma_{d}$, where $\sigma_{d}$ is the standard deviation of objects in the same class for 


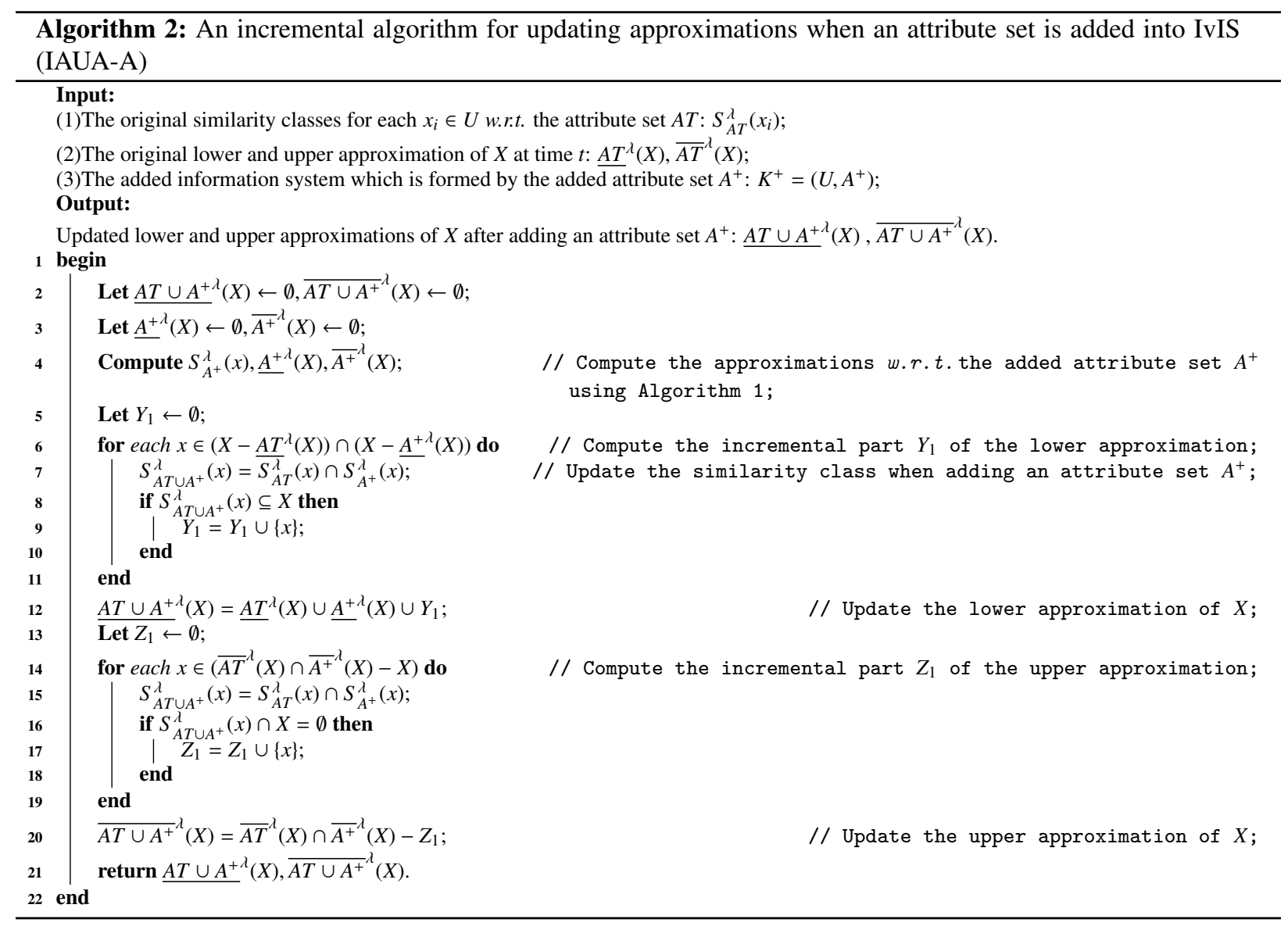

the attribute $a$. Then we design an interval-valued data generator to generate three different interval-valued data sets. The description of all above data sets is shown in Table 3. The experiments are all conducted on a PC with Inter(R) Core(TM)2 Quad CPU Q8400, 4.0 GB of memory and 32-bit Windows 7 OS. The algorithms are developed in C++ and the software is Dev-C++4.9.8.0.

Table 3: A description of data sets

\begin{tabular}{llcccc}
\hline & Data sets & Abbreviation & Instances & Attributes & Source \\
\hline 1 & Wine recognition data & Wine & 178 & 13 & UCI \\
2 & Wisconsin Diagnostic Breast Cancer & Wdbc & 569 & UCI & UCI \\
3 & Image Segmentation data & Segmentation & 2310 & 6118 & UCI \\
4 & First-order theorem proving & Prove & 9120 & UCI \\
5 & Daily and Sports Activities & Dasa & 10000 & 10 & Data generator \\
6 & Artificial data 1 & AD1 & 1000 & 200 & Data generator \\
7 & Artificial data 2 & AD2 & 1000 & Data generator \\
8 & Artificial data 3 & AD3 & & & 2000 \\
\hline
\end{tabular}

\subsection{Comparative experiments with the increasing size of data sets}

In this subsection, we compare the computational time of Algorithm IAUA-A (see Algorithm 2) and Algorithm IAUA-D (see Algorithm 3) with that of Algorithm SACA (see Algorithm 1) on the 8 data sets shown in Tabel 3. In order to better demonstrate the results of the experiments and analogy with other methods, we take out the first $10 \%, 20 \%, \ldots, 90 \%, 100 \%$ objects as ten different sizes of data sets for each of the eight data sets. They are separately 


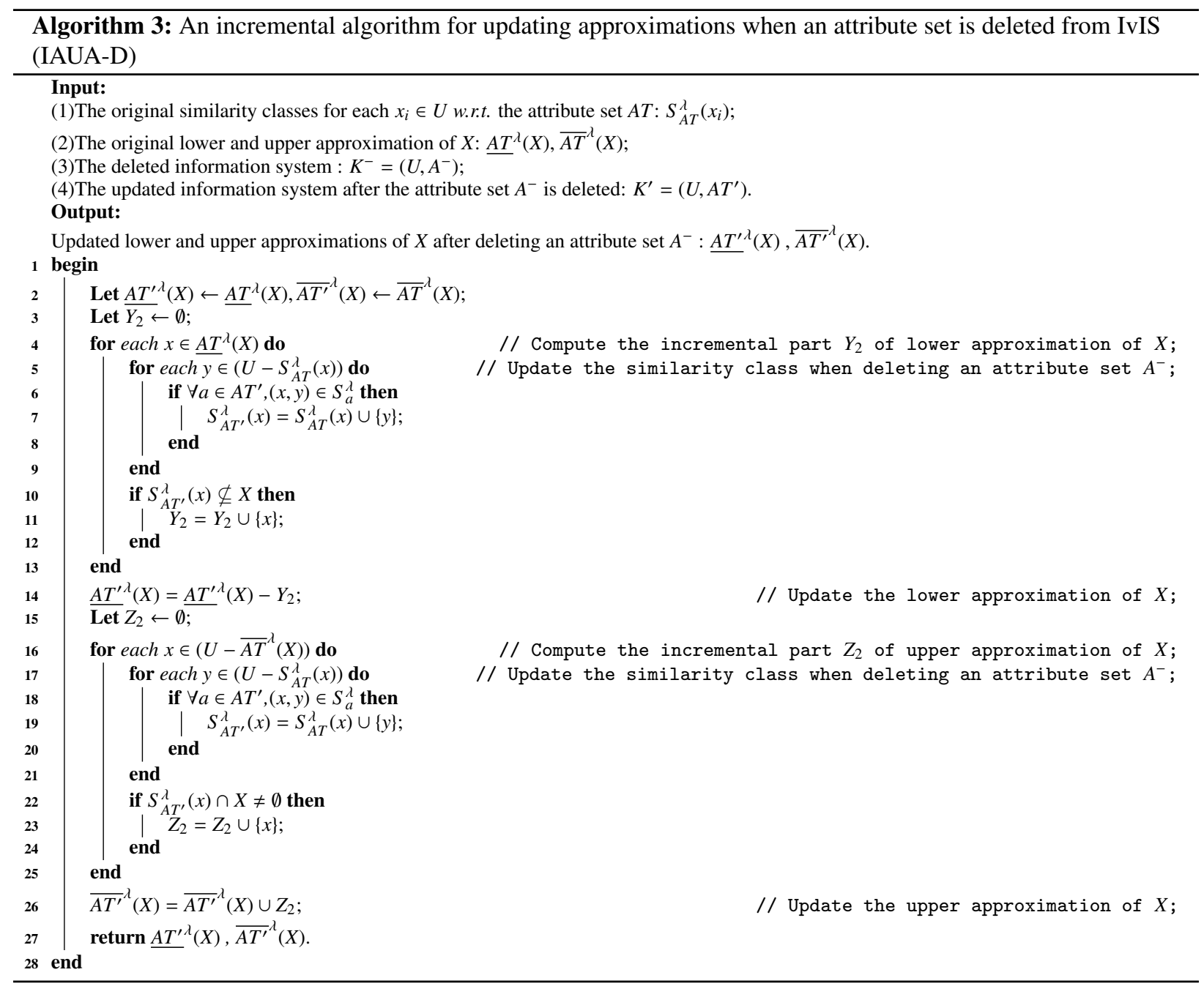

used to obtain the computational times by non-incremental and incremental algorithms and the results are shown as follows:

1. A comparison of SACA and IAUA-A

We compare Algorithm SACA with Algorithm IAUA-A on the eight data sets when adding an attribute set to the original attribute set. For each data set, the original attributes and the added attributes are listed in Table 4 . The comparative results are depicted in Figure 3 with the increasing size of the data set. In each of the subfigures (a)(h), the $\mathrm{x}$-coordinate pertains to the number of the data set (the 10 data sets starting from the smallest one), while $\mathrm{y}$-coordinate concerns the running-time for computing approximations. 
Table 4: A description of added attribute sets

\begin{tabular}{lllll}
\hline & Data sets & Attributes & Original attribute set & Added attribute set \\
\hline 1 & Wine & 13 & $\left\{a_{1}, a_{2}, \ldots, a_{9}\right\}$ & $\left\{a_{10}, a_{11}, a_{12}, a_{13}\right\}$ \\
2 & Wdbc & 30 & $\left\{a_{1}, a_{2}, \ldots, a_{25}\right\}$ & $\left\{a_{26}, a_{27}, \ldots, a_{30}\right\}$ \\
3 & Segmentation & 19 & $\left\{a_{1}, a_{2}, \ldots, a_{15}\right\}$ & $\left\{a_{16}, a_{17}, a_{18}, a_{19}\right\}$ \\
4 & Prove & 51 & $\left\{a_{1}, a_{2}, \ldots, a_{44}\right\}$ & $\left\{a_{45}, a_{46}, \ldots, a_{51}\right\}$ \\
5 & Dasa & 5625 & $\left\{a_{1}, a_{2}, \ldots, a_{5000}\right\}$ & $\left\{a_{5001}, a_{5002}, \ldots, a_{5625}\right\}$ \\
6 & AD1 & 10 & $\left\{a_{1}, a_{2}, \ldots, a_{7}\right\}$ & $\left\{a_{8}, a_{9}, a_{10}\right\}$ \\
7 & AD2 & 200 & $\left\{a_{1}, a_{2}, \ldots, a_{190}\right\}$ & $\left\{a_{191}, a_{192}, \ldots, a_{200}\right\}$ \\
8 & AD3 & 2000 & $\left\{a_{1}, a_{2}, \ldots, a_{1500}\right\}$ & $\left\{a_{1501}, a_{1502}, \ldots, a_{2000}\right\}$ \\
\hline
\end{tabular}
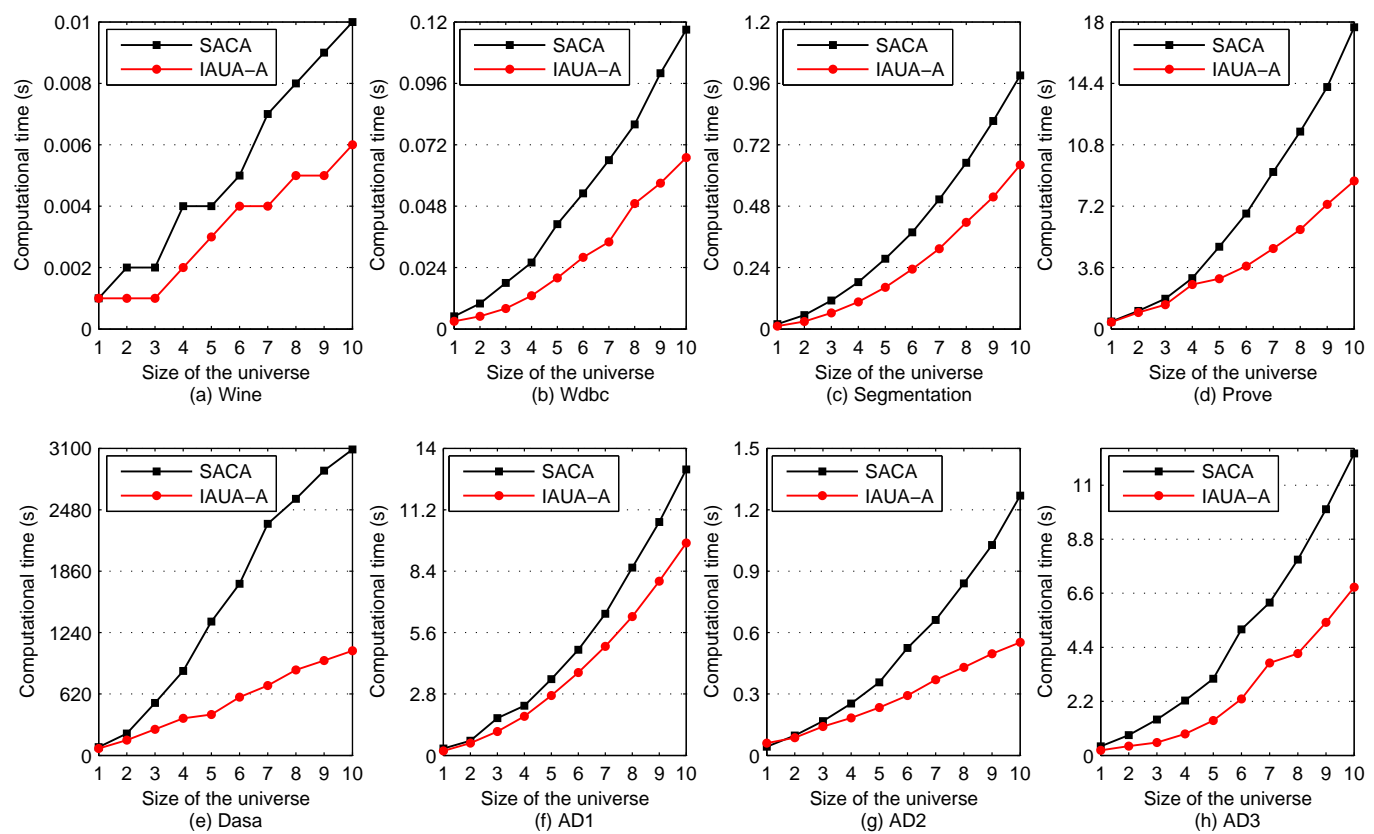

Figure 3: The comparison of computation time between Algorithm SACA and IAUA-A versus the size of data sets

From Figure 3, it is easy to see that the computational time of each of the two algorithms increases with the increasing size of data on all eight data sets. And according to the trend line of each of two algorithms, we see that Algorithm IAUA-A is always faster than Algorithm SACA on all the data sets even the high-dimensional data sets when adding the same attribute set to the original information systems. Furthermore, the difference becomes larger when the size of data sets increases.

2. A comparison of SACA and IAUA-D

We compare Algorithm SACA with Algorithm IAUA-D on the eight data sets when deleting an attribute set from the original attribute set. For each data set, the original attributes and the deleted attributes are listed in Table 5. The comparative results are depicted in Figure 4.

From Figure 4, it is also clear that the computational time of each of the two algorithms rises with the increasing size of the data. And Algorithm IAUA-D is always faster than Algorithm SACA on all the data sets even the highdimensional data sets when the same attribute set is deleted from the original attribute set in the IvIS. Furthermore, the difference becomes larger when the size of data sets increases in most of cases. 
Table 5: A description of deleted attribute set

\begin{tabular}{lllll}
\hline & Data sets & Attributes & Original attribute set & deleted attribute set \\
\hline 1 & Wine & 13 & $\left\{a_{1}, a_{2}, \ldots, a_{13}\right\}$ & $\left\{a_{10}, a_{11}, a_{12}, a_{13}\right\}$ \\
2 & Wdbc & 30 & $\left\{a_{1}, a_{2}, \ldots, a_{30}\right\}$ & $\left\{a_{20}, a_{21}, \ldots, a_{30}\right\}$ \\
3 & Segmentation & 19 & $\left\{a_{1}, a_{2}, \ldots, a_{19}\right\}$ & $\left\{a_{10}, a_{11}, \ldots, a_{19}\right\}$ \\
4 & Prove & 51 & $\left\{a_{1}, a_{2}, \ldots, a_{51}\right\}$ & $\left\{a_{45}, a_{46}, \ldots, a_{51}\right\}$ \\
5 & Dasa & 5625 & $\left\{a_{1}, a_{2}, \ldots, a_{5625}\right\}$ & $\left\{a_{5001}, a_{5002}, \ldots, a_{5625}\right\}$ \\
6 & AD1 & 10 & $\left\{a_{1}, a_{2}, \ldots, a_{10}\right\}$ & $\left\{a_{8}, a_{9}, a_{10}\right\}$ \\
7 & AD2 & 200 & $\left\{a_{1}, a_{2}, \ldots, a_{200}\right\}$ & $\left\{a_{191}, a_{192}, \ldots, a_{200}\right\}$ \\
8 & AD3 & 2000 & $\left\{a_{1}, a_{2}, \ldots, a_{2000}\right\}$ & \\
\hline
\end{tabular}
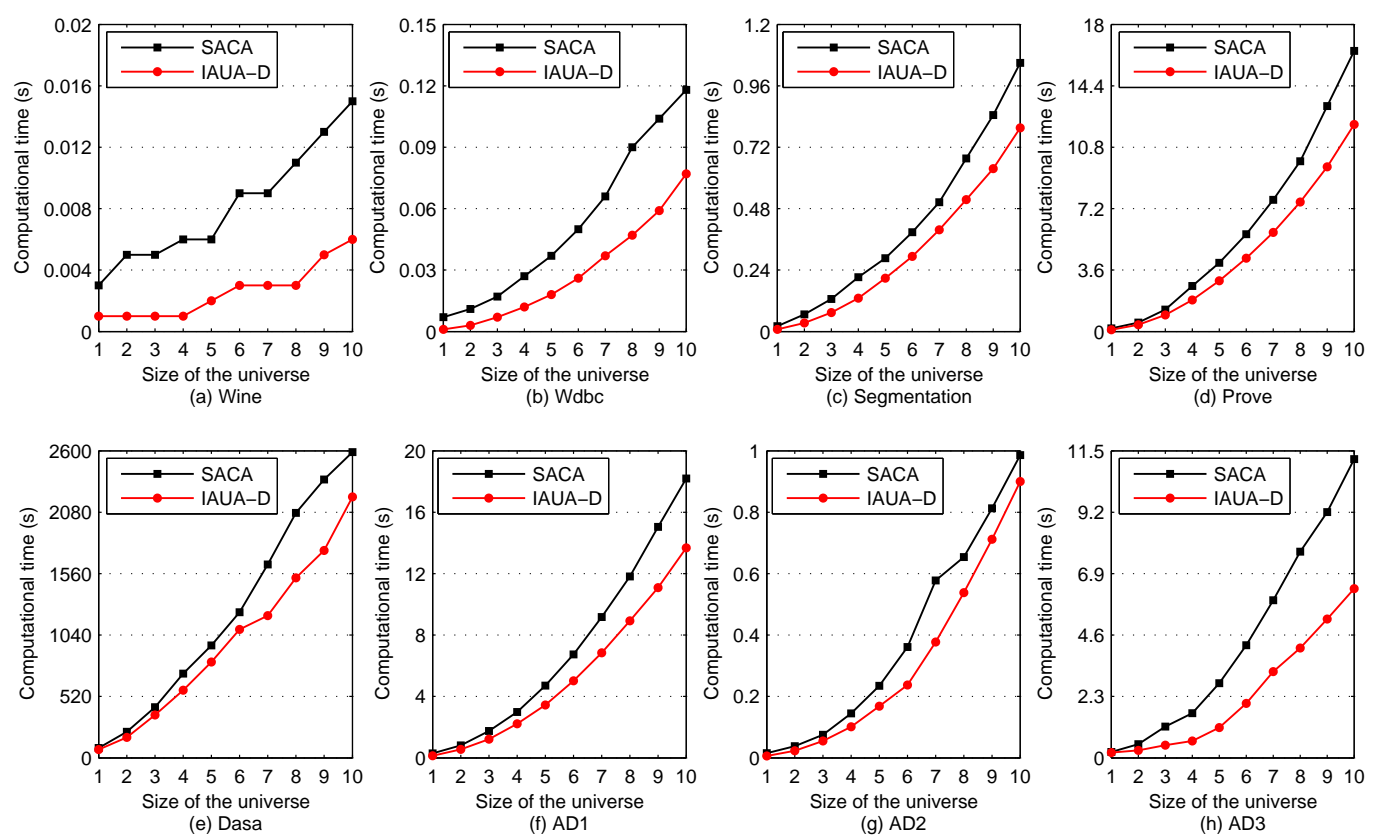

Figure 4: The comparison of computation time between Algorithm SACA and IAUA-D versus the size of data sets

\subsection{Comparative experiments with the increasing ratio of adding or deleting attributes}

In this subsection, we investigate the performance of the two incremental algorithms (Algorithm IAUA-A and Algorithm IAUA-D) when the ratio of the added or deleted attributes increases and the size of the objects keeps unchanged on two data sets with more attributes, which are First-order theorem proving (Prove) and Artificial data 2 (AD2).

For Algorithm IAUA-A, we take out the first $40 \%$ attributes of each of the two data sets as the original attribute sets. And then $10 \%, 20 \%, \ldots, 100 \%$ of the rest of attributes are added into them, respectively, while the size of objects keeps unchanged. The computational time of Algorithm SACA and Algorithm IAUA-A on updating approximations are obtained, respectively, and the results are depicted in Figure 5. In each subfigure of Figure 5, the X-coordinate pertains to the ratio of adding attributes which starts from $10 \%$ to $100 \%$ of the original attributes and the y-coordinate concerns the computation time of different algorithms. From Figure 5, it is clear that Algorithm IAUA-A is always faster than Algorithm SACA when the ratio of adding attributes increases. Therefore, Algorithm IAUA-A is efficient no matter what the ratio of adding attributes is. 

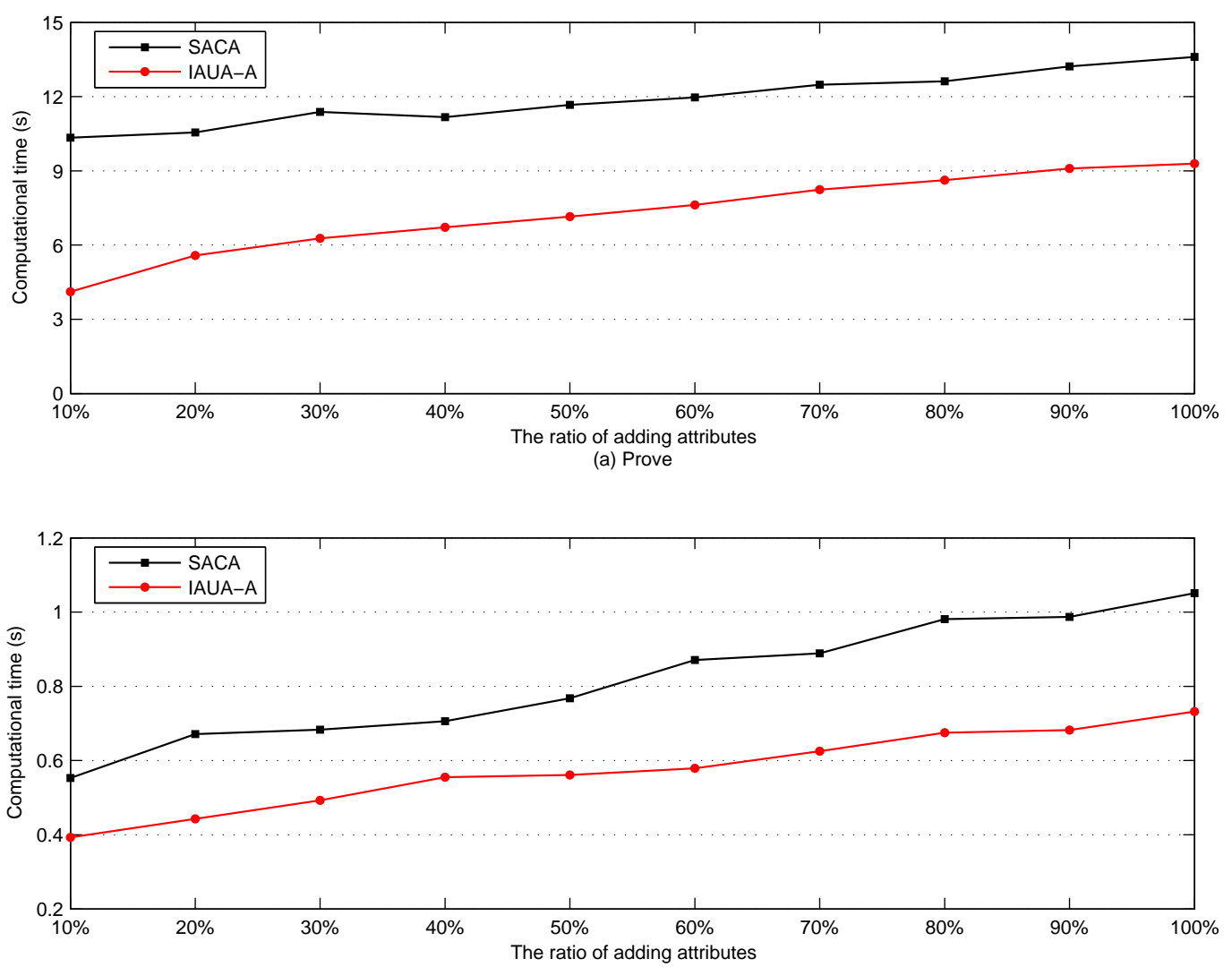

(b) AD2

Figure 5: The comparison of computation time between Algorithm SACA and IAUA-A versus the ratio of adding attributes

For Algorithm IAUA-D, we take out the whole attributes of each of the two data sets as the original attribute sets. And 10\%,20\%, . .,50\% attributes are deleted from them, respectively, while the size of objects keeps unchanged. Then the computational time of Algorithm SACA and Algorithm IAUA-D on updating approximations are obtained, respectively, and the results are depicted in Figure 6. In each subfigure of Figures 6, the x-coordinate pertains to the ratio of deleting attributes which starts from $10 \%$ to $50 \%$ of the original attributes and the y-coordinate concerns the computation time of different algorithms. In Figure 6(a) and Figure 6(c), we see that the computational time of Algorithm IAUA-D is a little less than or even as much as that of Algorithm SACA when the ratio of deleting attributes is less than 50\%. And in Figure 6(b) and Figure 6(d), we see that Algorithm IAUA-D is much faster than Algorithm SACA when the ratio of deleting attributes is less than 50\%. Clearly, Algorithm IAUA-D is influenced by the ratio of deleting attributes and the size of the concept $X$, which confirms the conclusion of time complexity analysis of Algorithm IAUA-D in Section 4. 

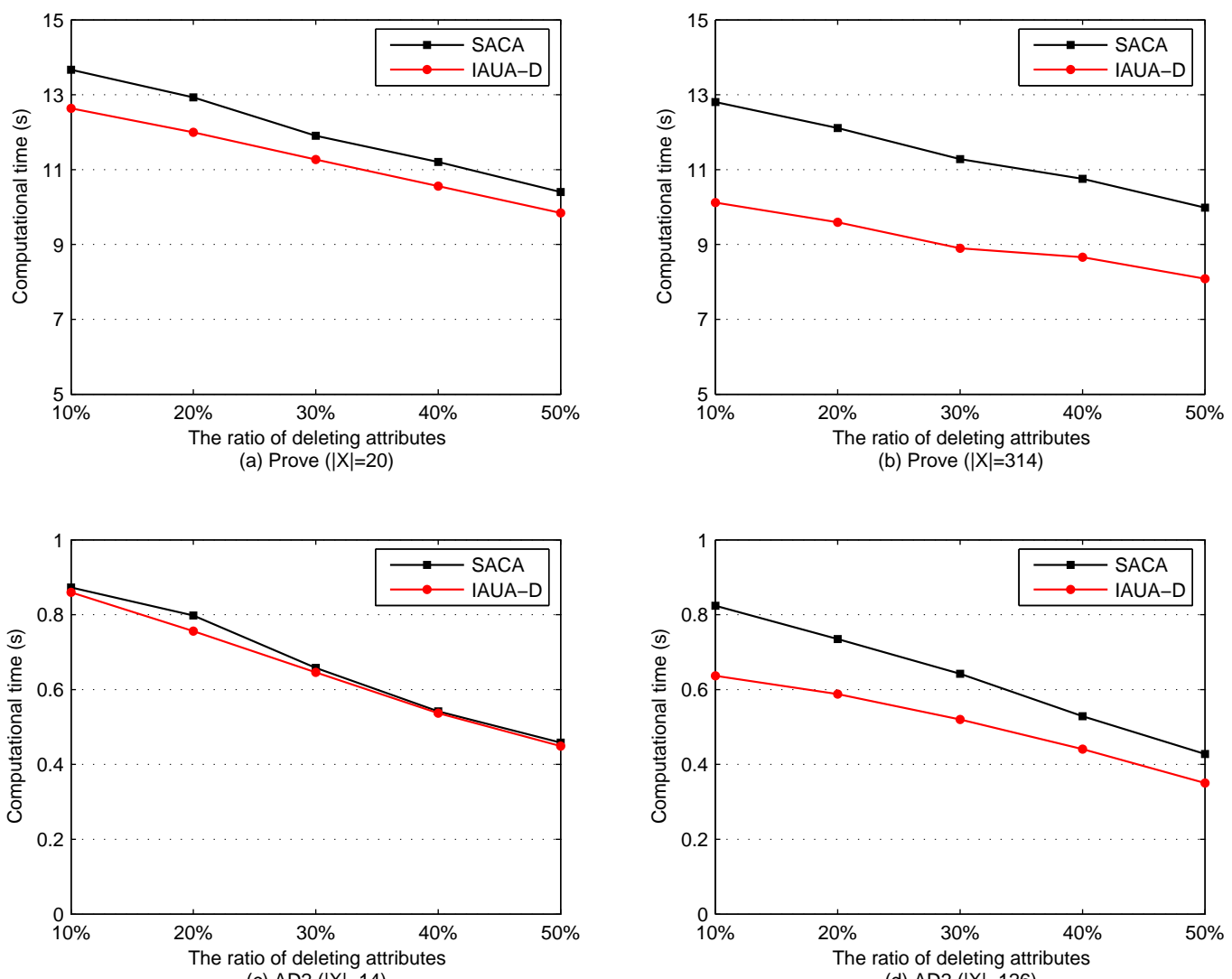

(c) AD2 (|X|=14)

(d) AD2 (|X|=126)

Figure 6: The comparison of computation time between Algorithm SACA and IAUA-D versus the ratio of deleting attributes

\section{Conclusion}

IvIS is usually used to describe uncertain problems in the real-life applications. And the attribute set in an IvIS always changes over time with the collection of new information. In this paper, by the similarity-based RST in IvIS, we proposed two incremental methods for updating rough approximations when adding and removing attributes, respectively. Two corresponding incremental algorithms for updating rough approximations were developed, respectively. A series of experiments on different preprocessed UCI data sets and artificial data sets were conducted to evaluate the performance of the proposed algorithms. The experimental results validated that the proposed incremental algorithms effectively reduce the computational time of updating approximations as opposed to the static (non-incremental) algorithm. Our future work will focus on incremental updating attribute reduction and decision rules when adding and removing attributes in interval-valued or real-valued decision systems, which is an interesting direction for dynamic knowledge discovery.

\section{Acknowledgements}

This work is supported by the National Science Foundation of China (Nos. 61573292, 61572406), the Young Software Innovation Foundation of Sichuan Province, China (No. 2014-046) and the Beijing Key Laboratory of Traffic Data Analysis and Mining (BKLTDAM2014001). 


\section{References}

[1] C.G. Bai, D. Dhavale, J. Sarkis, Complex investment decisions using rough set and fuzzy c-means: An example of investment in green supply chains. European Journal of Operational Research, 248(2)(2016) 507-521.

[2] U. Bentkowska, H. Bustince, A. Jurio, M. Pagola, B. Pekala, Decision making with an interval-valued fuzzy preference relation and admissible orders. Applied Soft Computing, 35(2015) 792-801.

[3] C. Blake, E. Keogh, C.J. Merz, UCI repository of machine learning databasesDepartment of Information and Computer Science, University of California, Irvine, CA $(1998)<$ http : //www.ics.uci.edu/ mlearn/MLRepository.html >

[4] Y. Chen, C. Cheng, A soft-computing based rough sets classifier for classifying IPO returns in the financial markets. Applied Soft Computing, 12(1)(2012) 462-475.

[5] Z.C. Chen, K.Y. Qin, Attribute reduction of interval-valued information system based on variable precision tolerance relation. Computer Science, 36(3)(2009) 163-166 (in Chinese).

[6] H.M. Chen, T.R. Li, C. Luo, S.J. Horng, G.Y. Wang. A decision-theoretic rough set approach for dynamic data mining. IEEE Transactions on Fuzzy Systems, 23(6)(2015) 1958-1970.

[7] J.H. Dai, W.T. Wang, J.S. Mi, Uncertainty measurement for interval-valued information systems. Information Sciences, 251(2013) 63-78.

[8] J.H. Dai, W.T. Wang, Q. Xu, H.W. Tian, Uncertainty measurement for interval-valued decision systems based on extended conditional entropy. Knowledge-Based Systems, 27(2012) 443-450.

[9] J. Derrac, F. Chiclanab, S. Garcłac, F. Herrera, Evolutionary fuzzy k-nearest neighbors algorithm using interval-valued fuzzy sets. Information Sciences, 329(2016) 144-163.

[10] W.S. Du, B.Q. Hu, Approximate distribution reducts in inconsistent interval-valued ordered decision tables. Information Sciences, 271(2014) 93-114.

[11] B. Huang, D.K. Wei, H.X. Li, Y.L. Zhuang, Using a rough set model to extract rules in dominance-based interval-valued intuitionistic fuzzy information systems. Information Sciences, 221(2013) 215-229.

[12] H.H. Inbarani, A.T. Azar, G. Jothi, Supervised hybrid feature selection based on PSO and rough sets for medical diagnosis. Computer Methods and Programs in Biomedicine, 1(2014) 175-185.

[13] C. Lauro, F. Gioia, Dependence and interdependence analysis for interval-valued variables. Data Science and Classification. Studies in Classification, Data Analysis, and Knowledge Organization, (2005) 171-183.

[14] Y. Leung, M.M. Fischer, W.Z. Wu, J.S. Mi, A rough set approach for the discovery of classification rules in interval-valued information systems. International Journal of Approximate Reasoning, 47(2)(2008) 233-246.

[15] T.R. Li, D. Ruan, W. Geert, J. Song, Y. Xu, A rough sets based characteristic relation approach for dynamic attribute generalization in data mining. Knowledge-Based Systems, 20(5)(2007) 485-494.

[16] S.Y. Li, T.R. Li, D. Liu, Incremental updating approximations in dominance-based rough sets approach under the variation of the attribute set. Knowledge-Based Systems, 40(2013) 17-26.

[17] D.C. Liang, D. Liu, Systematic studies on three-way decisions with interval-valued decision-theoretic rough sets. Information Sciences, 276(2014) 186-203.

[18] C. Luo, T.R. Li, H.M. Chen, D. Liu, Incremental approaches for updating approximations in set-valued ordered information systems. Knowledge-Based Systems, 50(2013) 218-233.

[19] C. Luo, T.R. Li, H.M. Chen, Dynamic maintenance of approximations in set-valued ordered decision systems under the attribute generalization. Information Sciences, 257(2014) 210-228.

[20] C. Luo, T.R. Li, H.M. Chen, L.X. Lu, Fast algorithms for computing rough approximations in set-valued decision systems while updating criteria values. Information Sciences, 299(2015) 221-242

[21] D.Q. Miao, N. Zhang, X.D. Yue, Knowledge reduction in interval-valued information systems, in: Proceedings of the 8th IEEE International Conference on Congitive Informatics (ICCI'09), 2009, 320-327.

[22] R.E. Moore, R.B. Kearfott and M.J. Cloud (Eds.), Introduction to Interval Analysis. Society for Industrial and Applied Mathematics, Philadelphia, (2009).

[23] R. Moore, W. Lodwick, Interval analysis and fuzzy set theory. Fuzzy Sets and Systems, 135(2003) 5-9.

[24] D.D. Nguyen, L.T. Ngo, L.T. Pham, W. Pedrycz, Towards hybrid clustering approach to data classification: Multiple kernels based intervalvalued Fuzzy C-Means algorithms. Fuzzy Sets and Systems, 279(2015) 17-39.

[25] Z. Pawlak, Rough sets. International Journal of Computer and Information Sciences, 11(1982) 341-356.

[26] Z. Pawlak, Information systems theoretical foundation. Information Systems, 3(6)(1981) 205-218.

[27] Y.H. Qian, J.Y. Liang, C.Y. Dang, Interval ordered information systems. Computers \& Mathematics with Applications, 56(8)(2008) 19942009 .

[28] D. Ramyachitra, M. Sofia, P. Manikandan, Interval-value based particle swarm optimization algorithm for cancer-type specific gene selection and sample classification. Genomics Data, 5(2015) 46-50.

[29] J.Y. Shyng, H.M. Shieh, G.H. Tzeng, An integration method combining rough set theory with formal concept analysis for personal investment portfolios. Knowledge-Based Systems, 23(6)(2010) 586-597.

[30] J.Y. Shyng, H.M. Shieh, G.H. Tzeng, S.H. Hsieh, Using FSBT technique with rough set theory for personal investment portfolio analysis. European Journal of Operational Research, 201(2)(2010) 601-607.

[31] B.Z. Sun, Z.T. Gong, D.G. Chen, Fuzzy rough set theory for the interval-valued fuzzy information systems. Information Sciences, 178(13)(2008) 2794-2815.

[32] T.B. Tseng, M.C. Jothishankar, T.T. Wu, Quality control problem in printed circuit board manufacturing: An extended rough set theory approach. Journal of Manufacturing Systems, 23(1)(2004) 56-72.

[33] Sh.P. Wan, D.F. Li, Fuzzy mathematical programming approach to heterogeneous multiattribute decision-making with interval-valued intuitionistic fuzzy truth degrees. Information Sciences, 325(2015) 484-503. 
[34] F.H. Wang, On acquiring classification knowledge from noisy data based on rough set. Expert Systems with Applications, 29(1)(2005) 49-64.

[35] J. Wu, F. Chiclana, A social network analysis trust-consensus based approach to group decision-making problems with interval-valued fuzzy reciprocal preference relations. Knowledge-Based Systems, 59(2014) 97-107.

[36] T. Xiong, Y.K. Bao, Zh.Y. Hu, R. Chiong, Forecasting interval time series using a fully complex-valued RBF neural network with DPSO and PSO algorithms. Information Sciences, 305(2015) 77-92.

[37] X.B. Yang, D.J. Yu, J.Y. Yang, L.H. Wei, Dominance-based rough set approach to incomplete interval-valued information system. data \& knowledge engineering, 68(11)(2009) 1331-1347.

[38] X.B. Yang, Y. Qi, D.J. Yu, H.L. Yu, J.Y. Yang, $\alpha$-dominance relation and rough sets in interval-valued information systems. Information Sciences, 294(2015) 334-347.

[39] J. Yao, J.P. Herbert, Financial time-series analysis with rough sets. Applied Soft Computing, 9(3)(2009) $1000-1007$.

[40] Y.Y. Yao, Interval-set algebra for qualitative knowledge representation, in: Proceedings of the 5th International Conference Computing and Information (ICCI'93), 1993, 370-374.

[41] Y.Y. Yao, X. Li, Comparison of rough-set and interval-set models for uncertain reasoning, Fundamenta Informaticae, 27(1996) $289-298$.

[42] Y.Y. Yao, Q. Liu, A generalized decision logic in interval-set-valued information tables. Lecture Notes in Computer Science, 1711(1999) 285-293.

[43] A.P. Zeng, T.R. Li, D. Liu, J.B. Zhang, H.M. Chen, A fuzzy rough set approach for incremental feature selection on hybrid information systems. Fuzzy Sets and Systems, 258(2015) 39-60.

[44] X. Zhang, C.L. Mei, D.G. Chen, J.H. Li, Multi-confidence rule acquisition and confidence-preserved attribute reduction in interval-valued decision systems. International Journal of Approximate Reasoning, 55(8)(2014) 1787-1804.

[45] H.Y. Zhang, Y. Leung, L. Zhou, Variable-precision-dominance-based rough set approach to interval-valued information systems. Information Sciences, 244(2013) 75-91.

[46] J.B. Zhang, T.R. Li, H.M. Chen, Composite rough sets for dynamic data mining. Information Sciences, 257(2014) 81-100.

[47] J.B. Zhang, Y. Zhu, Y. Pan, T.R. Li, Efficient parallel boolean matrix based algorithms for computing composite rough set approximations. Information Sciences, 329(2016) 287-302.

[48] Y.Y. Zhang, T.R. Li, C. Luo, H.M. Chen, Incremental updating rough approximations in interval-valued information systems, in: Proceedings of the 10th International Conference on Rough Sets and Knowledge Technology (RSKT2015), 2015, $243-252$.

[49] H.Y. Zhang, W.X. Zhang, W.Z. Wu, On characterization of generalized interval-valued fuzzy rough sets on two universes of discourse. International Journal of Approximate Reasoning, 51 (2009) 56C70.

[50] X.R. Zhao, B.Q. Hu, Fuzzy and interval-valued fuzzy decision-theoretic rough set approaches based on fuzzy probability measure. Information Sciences, 298(2015) 534C554.

[51] B.Q. Hu, Generalized interval-valued fuzzy variable precision rough sets determined by fuzzy logical operators. International Journal of Fuzzy Systems, 16(4)(2014) 554-565.

[52] T. Zhao, J. Xiao, J.L. Ding, P. Chen, A variable precision interval type-2 fuzzy rough set model for attribute reduction. International Journal of Fuzzy Systems, 26(6)(2014) 2785-2797. 